\title{
IDENTIFICAÇÃO DE INDICADORES DE QUALIDADE PARA UM SERVIÇO DE TRANSPLANTE RENAL
}

\author{
Identification of quality indicators to monitoring of a renal transplant service
}

Patrícia Treviso', Flávio Henrique Brandão², David Saitovitch ${ }^{3}$.

\begin{abstract}
RESUMO
Objetivo: Identificar os principais indicadores de qualidade para um Serviço de Transplante Renal, na opinião de profissionais e pacientes. Método: Estudo exploratório, descritivo, análise quantitativa, coleta de dados através de questionário estruturado com perguntas abertas e fechadas. Amostra $(\mathrm{n}=133)$ composta por profissionais assistenciais, administrativos e pacientes. Resultados: Não houve diferença significativa quanto à importância dos indicadores apresentados, na opinião dos profissionais. A diferença estatística foi observada em: necessidade de mais indicadores, na opinião dos profissionais assistenciais $(\mathrm{p}=0,046)$ e na opinião dos profissionais administrativos $(\mathrm{p}=0,011)$. Os pacientes enfatizaram a necessidade de indicadores relacionados à humanização do cuidado. Conclusão: Os indicadores institucionais atuais a nível hospitalar são insuficientes e não contemplam a realidade atual de um programa de transplante. Este trabalho possibilitou a identificação de uma lista consistente de 23 indicadores específicos, que podem ser utilizados pelos Serviços de Transplante Renal.
\end{abstract}

Descritores: Indicadores; Indicadores de Qualidade em Assistência à Saúde; Transplante Renal; Garantia da Qualidade dos Cuidados de Saúde, Controle de Qualidade

\section{Instituições:}

${ }^{1}$ Programa de Pós Graduação de Medicina e Ciências da Saúde - Nefrologia. Pontifícia Universidade Católica do Rio Grande do Sul. Porto Alegre/RS

${ }^{2}$ Departamento de Administração, Instituto Brasileiro de Gestão em Negócios, Porto Alegre/RS

${ }^{3}$ Departamento de Medicina Interna. Faculdade de Medicina. Pontifícia

Universidade Católica do Rio Grande do Sul - Porto Alegre/RS

Correspondência:

Patrícia Treviso

Rua Dr Tauphick Saadi, 33/601 - Porto Alegre / RS - Cep 90470-040

Fone: (51) 9644-1456

E-mail: ptreviso@ibest.com.br

Recebido em: 16.11 .2009

\section{INTRODUÇÃO}

A busca constante pela qualidade na prestação de serviços tornouse pré-requisito para a sobrevivência e competitividade das instituições. ${ }^{1-2}$ Nesse contexto, a adoção de um sistema de gestão da qualidade de serviços de saúde é uma decisão estratégica além de uma necessidade social e técnica, entendendo que a melhoria da qualidade leva diretamente à maior eficiência e redução de custos. ${ }^{2-7}$

A definição de qualidade segundo a literatura ${ }^{8}$ é que a qualidade aumenta o grau de chance dos serviços de saúde atingirem os desfechos desejados. Portanto, as chances das instituições de saúde manterem-se no mercado estão ligadas diretamente aos processos de melhoria contínua da qualidade e é baseado nessa hipótese que este trabalho se desenvolve, acreditando que os serviços de transplante renal podem dispor de ferramentas para se tornar ainda mais qualificados, sendo uma dessas ferramentas os indicadores de qualidade.

Indicador de qualidade é uma ferramenta desenvolvida para facilitar a quantificação e avaliação das informações, ${ }^{9}$ sendo utilizada para medir a qualidade em um aspecto concreto da atenção à saúde, podendo auxiliar no monitoramento de processos e seus resultados, bem como fornecer informações críticas sobre a eficácia destes. ${ }^{7,10}$

$\mathrm{O}$ estudo proposto, que consideramos a primeira abordagem dessa temática ainda não estudada em profundidade na área de transplantes, objetiva identificar os principais indicadores de qualidade para um Serviço de Transplante Renal na opinião de profissionais e pacientes, podendo ser útil como instrumento para uma gestão baseada na melhoria da qualidade. 


\section{MÉTODOS}

Estudo contemporâneo transversal com análise quantitativa, realizado no Hospital São Lucas da PUCRS. A coleta de dados deu-se através de questionário estruturado com perguntas abertas e fechadas, diferenciado para profissionais e pacientes e efetuado no mês de dezembro de 2007. Amostragem aleatória, descrita na Tabela 1.

Tabela 1 - Caracterização da amostra $(n=133)$

\begin{tabular}{|l|c|c|}
\hline \multicolumn{2}{|l|}{ Variável } & \multicolumn{2}{|l|}{ Amostra: $n(\%)$} & Universo (n) \\
\hline Categorias profissionais & $10(71,0)$ & 14 \\
\hline Líderes administrativos & $6(100,0)$ & 6 \\
\hline Auxiliares administrativos & $13(100,0)$ & 13 \\
\hline Médicos & $23(72,0)$ & 32 \\
\hline Enfermeiras & $20(12,0)$ & 169 \\
\hline Técnicos de enfermagem & $21(7,0)$ & 297 \\
\hline Pacientes pré-transplantados & $40(12,7)$ & 315 \\
\hline Pacientes pós-transplantados & $133(15,7)$ & 846 \\
\hline Total & & \\
\hline
\end{tabular}

Nota: Os dados são apresentados como freqüência ou percentual.

Todos os profissionais atuantes na área assistencial ou administrativa do Serviço de Transplante Renal do HSL-PUCRS foram convidados a participar do estudo no período da coleta, bem como os pacientes que estavam internados ou realizaram consulta no ambulatório naquele período, tendo sido incluídos os que atenderam os aspectos éticos.

Após revisão da literatura, conversas com expertises na área de qualidade, diretores e/ou responsáveis pela gestão da qualidade de diferentes empresas, da área da saúde e outras, observamos que existem diferentes maneiras de se construir uma grade de indicadores para um serviço específico. No presente trabalho, resolvemos questionar os envolvidos com transplante renal: profissionais e pacientes. Com base nos indicadores do SIPAGEH (Sistema de Indicadores Padronizados para a Gestão Hospitalar) e em indicadores institucionais da PUCRS, organizou-se uma lista de indicadores gerais (assistenciais e administrativos) que se aplicariam ao serviço, lista essa que foi submetida à avaliação dos sujeitos através do questionário. Os indicadores foram pontuados de 1 a 5, conforme escala de Lickert. Para tabulação dos dados, essa pontuação foi dividida em dois grupos: não importantes (respostas 1 a 3) e importantes (respostas 4 e 5). Considerou-se também a sugestão de outros indicadores necessários para tal serviço, através de perguntas abertas.

Características de inclusão: maiores de 18 anos; alfabetizados, pacientes em lista de espera ou que já realizaram transplante renal (doador falecido); profissionais atuantes no processo de transplante renal.

O início do estudo ocorreu após avaliação e emissão do Parecer Consubstanciado de Aprovação pelo Comitê de Ética e Pesquisa da PUCRS. Foram incluídos no estudo somente os indivíduos que concordaram e assinaram o Termo de Consentimento Livre e Esclarecido. O nível de significância adotado foi $\mathrm{p} \leq 0,05$. Para esses cálculos, usou-se o programa Statistical Package for Social Sciences (SPSS, versão 15 para Windows, SPSS Inc, Chicago, IL, EUA).

\section{RESULTADOS}

Foram incluídos no estudo 133 (100\%) sujeitos, sendo que destes, $72(54,14 \%)$ eram profissionais assistenciais e administrativos, e $61(45,86 \%)$ eram pacientes pré- e pós-transplante. Em relação às características demográficas dos participantes do estudo, $83(62,4 \%)$ eram do sexo feminino e com idade média de 40,3 $\pm 13,1$. Com relação à população de nível superior, $44,6 \%$ estavam distribuídas entre os profissionais administrativos e assistenciais e 1,6\% entre os pacientes. Em relação ao tempo de atuação em transplante, 25\% dos profissionais atuavam há mais de 10 anos nessa área. Entre os pacientes, $65 \%$ eram transplantados, dos quais $80 \%$ correspondiam a pacientes ambulatoriais.

Os indicadores assistenciais analisados pelos profissionais assistenciais foram: Índice de infecção hospitalar, taxa de mortalidade, tempo de permanência (Unidade de Internação/Unidade de Tratamento Intensivo), complicações cirúrgicas, complicações pós-transplante, intercorrências assistenciais, índice de reinternações, satisfação cliente interno, satisfação cliente externo.

Através da análise dos dados, observou-se que tanto para os técnicos, como para os enfermeiros e médicos, os indicadores assistenciais tiveram pesos relativamente similares. $\mathrm{O}$ indicador “complicações cirúrgicas" obteve maior pontuação, tendo apenas um indivíduo considerado não importante e o indicador "satisfação do cliente interno" foi o indicador com menor pontuação, tendo sido considerado não-importante por sete profissionais, não obstante todos indicadores foram considerados importantes numa taxa superior a $80 \%$, tendo na maioria das vezes taxa de $90 \%$ ou maior.

Os indicadores analisados pelos profissionais administrativos foram: Índice de acidentes de trabalho, horas de treinamento, tempo de permanência (Unidade de Internação e Unidade de Tratamento Intensivo), taxa de ocupação, receita, custo, absenteísmo, satisfação cliente interno, satisfação cliente externo.

Os dados foram comparados entre os profissionais administrativos (auxiliar e líder administrativo). Não houve diferença estatística entre as categorias profissionais pesquisadas. $\mathrm{O}$ indicador "satisfação do cliente interno" foi considerado importante numa taxa de $100 \%$ pelas duas categorias profissionais; o indicador "índice de acidentes de trabalho" foi o único considerado não importante ou indiferente pela maioria dos sujeitos. Todos os outros indicadores foram considerados importantes, numa taxa igual ou superior a $60 \%$, tendo na maioria das vezes taxa de importância de 100\%. 
Houve diferença estatística observada em relação à necessidade de mais indicadores na opinião dos profissionais assistenciais $(p=0,046)$ e na opinião dos profissionais administrativos $(p=0,011)$. Pacientes enfatizaram a necessidade de indicadores relacionados à humanização do cuidado.

Através das perguntas abertas obteve-se 227 citações, resultando numa lista de 50 novos indicadores sugeridos pelos profissionais e pacientes; destes, 27 são indicadores institucionais e apenas 23 são específicos para um Serviço de Transplante Renal, os quais estão destacados na Tabela 2.

Tabela 2 - Listagem dos indicadores específicos sugeridos pelos profissionais assistenciais, administrativos e pacientes.

\begin{tabular}{|c|c|}
\hline $\mathbf{N}^{\circ}$ & Indicadores Sugeridos \\
\hline 1 & Tempo em lista de espera \\
\hline 2 & Número de doadores \\
\hline 3 & Divulgação e Marketing focado na doação \\
\hline 4 & Sobrevida do enxerto \\
\hline 5 & Índice de retorno à diálise \\
\hline 6 & Índice de rejeição do órgão \\
\hline 7 & Índice de mortalidade do paciente pós-transplante \\
\hline 8 & Índice de complicações pós-transplante \\
\hline 9 & Sobrevida do receptor \\
\hline 10 & Índice de falência do órgão \\
\hline 11 & Número de transplantes realizados \\
\hline 12 & Tempo de diálise pré-transplante e tipo de diálise \\
\hline 13 & Uso de hemoderivados no processo de transplante \\
\hline 14 & Reinternação pós-transplante \\
\hline 15 & Número de pacientes em lista de espera \\
\hline 16 & Perda primária do enxerto \\
\hline 17 & Motivo falta de doadores \\
\hline 18 & Função do enxerto nos 6 e 12 meses \\
\hline 19 & Tempo de isquemia fria do órgão \\
\hline 20 & Mortalidade em lista de espera \\
\hline 21 & Retorno à atividade profissional pós-transplante \\
\hline 22 & Número de atendimentos ambulatoriais pós-transplante \\
\hline 23 & Tipo de terapia / tratamento pré-transplante \\
\hline
\end{tabular}

Os indicadores relacionados à assistência somaram o maior número de citações, ressaltando-se o indicador institucional relacionado à agilidade, capacitação e humanização do cuidado.

\section{DISCUSSÃO}

Tradicionalmente, os hospitais utilizam indicadores gerais ou institucionais, os quais possibilitam avaliar a instituição na sua totalidade, porém não considerando a especificidade dos serviços. No entanto, a especificidade e a complexidade dos serviços de saúde exigem indicadores específicos. ${ }^{10}$

Os indicadores administrativos, como por exemplo: horas de treinamento, absenteísmo e custo tiveram uma taxa de importância que oscilou entre 33,3 e $100 \%$, sendo que os auxiliares pontuaram alguns indicadores como não importantes, e mesmo assim, não consideraram necessário incluir novos; já os líderes consideraram todos indicadores importantes e mesmo assim acharam importante incluir outros.

$\mathrm{Na}$ lista de indicadores sugeridos, os profissionais administrativos citaram itens relativos à assistência e não só às questões administrativas, o mesmo acontecendo com os profissionais assistenciais, demonstrando a ampla visão que estes profissionais têm dos processos desenvolvidos dentro da instituição, observando além do foco de sua atuação, avaliando o todo: a estrutura, os processos e os resultados. ${ }^{11}$

A lista de indicadores sugeridos corresponde a 227 citações, onde a maioria deles é assistencial, isto é, que se reportam a aspectos da assistência no macro processo do transplante renal, salientando-se a assistência multidisciplinar, agilidade e humanismo, demonstrando prioridade da assistência sobre as questões administrativas.

O número de indicadores obtidos neste estudo é grande, o que implica em dificuldade de colocar todos em uso; a literatura menciona que não há necessidade de muitos indicadores, mas sim de utilizar os que são mais adequados a cada instituição. Os resultados deste estudo permitem oferecer um leque de indicadores específicos, dos quais diferentes serviços de transplante renal poderão fazer uso selecionando os indicadores mais adequados para a realidade da instituição em que atuam.

Não há um número exato de indicadores necessários para o controle da qualidade de um serviço, porém, é importante que exista um grupo onde a coleta de dados e a análise possam ser viáveis, ${ }^{12,13} \mathrm{e}$ que esses dados possam ser úteis para tal serviço. Com base nisso, acredita-se que os Serviços de Transplante Renal possam eleger e usufruir dos indicadores elencados neste estudo, contribuindo assim para a melhoria de seus processos.

\section{CONCLUSÕES}

O presente estudo identificou os principais indicadores de qualidade para um Serviço de Transplante Renal com base na opinião de pacientes e profissionais, resultando numa lista de 50 indicadores, sendo 23 específicos para esse tipo de serviço.

Os profissionais assistenciais consideraram importantes todos os indicadores avaliados. Os profissionais administrativos demonstraram divergência de opiniões, contudo, consideraram a maioria dos indicadores administrativos importante.

Os indicadores institucionais atuais a nível hospitalar são insuficientes e não contemplam a realidade atual de um programa de transplante renal. Acreditamos que os indicadores específicos sugeridos neste estudo por profissionais e pacientes possam ser úteis no gerenciamento de qualidade para Serviços de Transplante Renal. 


\section{ABSTRACT}

Purpose: To identify major quality indicators of a Renal Transplant Service, according to the professionals and patients' opinion. Method: Exploratory and descriptive study, quantitative analysis, information gathered through an aimed questionnaire with open and close questions. Sample $(n=133)$ consisted by assistant and administrative professionals and by patients. Result: There was no relevant difference concerning the importance of the indicators presented, according to the professionals' opinion. It was observed a statistic difference as to: the need of additional indicators, according to the assistant professionals $(p=0,046)$ and the administrative professionals' opinion $(p=0,011)$. Patients emphasized the need of indicators related to the humanization of the care. Conclusion: The present institutional indicators in hospitals are insufficient and they do not comply with the present reality of a transplant program. This study allowed the identification of a consistent list of 23 specific indicators that can be used by Renal Transplant Services.

Keywords: Indicators; Quality Indicators, Health Care; Renal Transplant; Quality Control, Health Care; Quality Assurance.

\section{REFERÊNCIAS}

1. Manfredi SR. Implantação e Aplicação Prática do Programa de Melhoria Contínua da Qualidade em uma Unidade Satélite de Hemodiálise [tese]. São Paulo:Universidade Federal de São Paulo; 2000.

2. Schout D, Novaes HMD. Do registro ao indicador: gestão da produção da informação assistencial nos hospitais. Ciênc Saúde Coletiva. 2007;12:935-44.

3. Turchi LM. Texto para Discussão n. 459. Qualidade Total: Afinal de que estamos falando?. Brasília: Instituto de Pesquisa Econômica Aplicada - IPEA; 1997.

4. Carvalho COM.. Desenvolvimento de um modelo de gestão da qualidade em um serviço de hemodiálise [tese]. São Paulo: Universidade Federal de São Paulo; 2004.

5. Campos VF. Gerenciamento da rotina do trabalho do dia-a-dia. Belo Horizonte: Editora de Desenvolvimento Gerencial; 1998.

6. Souza C. Clientividade. Exame. 2000;710:184-6.

7. Fernandes DR. Uma contribuição sobre a construção de indicadores e sua importância para a gestão empresarial. Rev FAE 2004;7:1-18.

8. Institute of Medicine of the National Academies. Crossing the Quality Chasm: The
IOM Health Care Quality Initiative. [cited 2008 Sep 04] Available from: http://www. iom.edu/CMS/8089.aspx.

9. Organização Pan-Americana de Saúde. Indicadores básicos de saúde no Brasil: conceitos e aplicações / Rede Interagencial de Informações para a Saúde - RIPSA. Brasília: Organização Pan-Americana de Saúde, 2002.

10. United Nations General Assembly Special Session on HIV/AIDS. Monitoring the Declaration of Commitment on HIV/AIDS: Guidelines on construction of core indicators: 2008 reporting. Geneva: UNAIDS; 2007

11. Donabedian A. The criteria and standards of quality. In: Donabedian A. Explorations in Quality Assesment and Monitoring. Ann Arbor: Health Administration Press; 1982.

12. Van Waeleghem JP, Edwards P. European Standards for Nephrology Nursing Practice. Switzerland: European Dialysis and Transplantation Nurses Association - European Renal Care Association; 1995.

13. Kluck M. Indicadores de Qualidade para Assistência Hospitalar. [Acesso em dez. 2007]. Disponível em: http://www.cih.com.br/Indicadores.htm. 